Écrire

l'histoire

\section{Écrire l'histoire}

Histoire, Littérature, Esthétique

\section{3 | 2009}

Le détail (1)

\title{
Critique policière et recherche historique
}

Autour des livres de Pierre Bayard

\section{Sylvain Venayre}

\section{OpenEdition}

Journals

Édition électronique

URL : http://journals.openedition.org/elh/970

DOI : $10.4000 /$ elh. 970

ISSN : 2492-7457

Éditeur

CNRS Éditions

\section{Édition imprimée}

Date de publication : 1 juin 2009

Pagination : 104-107

ISBN : 978-2-35698-008-3

ISSN : 1967-7499

Référence électronique

Sylvain Venayre, "Critique policière et recherche historique », Écrire l'histoire [En ligne], 3 | 2009, mis en ligne le 01 juin 2012, consulté le 23 septembre 2020. URL : http://journals.openedition.org/elh/970 ; DOI : https://doi.org/10.4000/elh.970

Ce document a été généré automatiquement le 23 septembre 2020.

Tous droits réservés 


\title{
Critique policière et recherche historique
}

\author{
Autour des livres de Pierre Bayard
}

Sylvain Venayre

1 Avant de devenir, en 2007, l'auteur mondialement connu de Comment parler des livres que l'on n'a pas lus, Pierre Bayard, psychanalyste et professeur de littérature à l'université Paris VIII, s'était proposé d'être l'inventeur d'un genre littéraire. Mêlant réflexion critique, enquête policière et psychanalyse, il avait même défini un nouveau champ du savoir : la « critique policière ».

2 Toute nouvelle discipline devant s'enorgueillir de glorieux précurseurs, Pierre Bayard a trouvé le sien en la personne de Voltaire, dont les remarques sarcastiques sur l'invraisemblance de l'intrigue d' $\subset$ dipe roi de Sophocle ont traversé les siècles. De la même façon que Voltaire avait critiqué les incohérences du récit du meurtre de Laïos, Pierre Bayard a donc entrepris de relire certaines grandes enquêtes policières de l'histoire de la littérature afin d'en mettre au jour les contradictions.

Davantage: loin de s'en tenir au confort de l'attitude strictement dénonciatrice de Voltaire, Pierre Bayard a fait l'effort de rétablir chaque fois la vérité, c'est-à-dire de laver la mémoire des innocents injustement accusés par les auteurs de ces histoires et de démasquer les véritables assassins. Après avoir opéré ainsi avec Le Meurtre de Roger Ackroyd d'Agatha Christie, puis, de manière peut-être moins convaincante ${ }^{1}$, avec Hamlet, il a récemment relu de la sorte Le Chien des Baskerville d'Arthur Conan Doyle.

4 Ce dernier opus de la «critique policière» est une réussite. Pierre Bayard a magistralement pointé l'incroyable accumulation des erreurs de raisonnement de Sherlock Holmes lors de son enquête sur la lande de Dartmoor. Lavant le pauvre Stapleton de toutes les accusations portées contre lui, il a identifié le véritable meurtrier de Henry de Baskerville. Surtout, il a suggéré les raisons de l'aveuglement de Holmes. Furieux d'avoir été contraint de ressusciter son héros sous la pression du public, Conan Doyle aurait en effet conçu envers lui une haine telle que, plus ou moins consciemment, il aurait désiré ridiculiser le célèbre détective. De fait, aucun lecteur ne 
peut plus, raisonnablement, admirer la légendaire puissance de déduction de Sherlock Holmes après la démonstration de Pierre Bayard.

5 À première vue, rien n'est plus éloigné de la démarche de l'historien que les principes qui définissent le genre de la " critique policière ». Depuis la fin du XIX siècle, on le sait, l'histoire, en tant que discipline universitaire aspirant à un statut scientifique, s'est précisément construite contre la figure de l'historien «chargé de la vengeance des peuples " chère à Chateaubriand. Même s'il serait facile de prouver que, dans la pratique, les historiens peinent à se dégager d'un tel modèle, aucun d'entre eux n'accepterait de se reconnaître pleinement dans la figure de l'enquêteur de Pierre Bayard, soucieux de traquer les erreurs judiciaires passées, de réhabiliter les innocents injustement accusés et de dénoncer les coupables impunis.

Il y a pourtant, dans les séduisants paradoxes de l'inventeur de la « critique policière ", quelques invitations à réfléchir pour les chercheurs d'aujourd'hui, à commencer par les historiens. Le goût du jeu, par exemple, qui anime Pierre Bayard et donne à ses livres leur ton amusant et leur pouvoir de séduction, gagnerait ainsi à être partagé par des chercheurs qui, trop souvent, le condamnent dans le cadre de leur activité professionnelle.

7 Rappelons à ce propos que le jeu n'est pas lui-même le but de la démarche de Bayard. Innocentant des héros de papier voués depuis des décennies, voire des siècles, aux gémonies des lecteurs, démontrant la culpabilité d'autres personnages qui, jusqu'à présent, avaient échappé au légitime mépris du public, Bayard vise en réalité un autre objectif : l'élaboration d'une théorie littéraire et psychanalytique, laquelle, reposant sur le " principe d'incomplétude » et la notion d'« univers troué », fait du lecteur le centre de toute activité littéraire. Faute de compétences adéquates, on ne jugera pas ici de la pertinence de cette construction dans laquelle le spécialiste pourrait peut-être reconnaître, derrière l'ironie, des théories de la réception plus anciennes, jusqu'à voir dans la «critique policière » la queue de comète, colorée et drolatique, de l'« ère du soupçon ».

8 On retiendra, en revanche, la fonction assignée, dans l'élaboration de cette théorie, au jeu. Les chercheurs, nous suggère Pierre Bayard, n'ont rien à gagner à dissimuler la dimension ludique de leur activité. Non seulement le plaisir, la joie augmentent l'intensité et la qualité du travail fourni, mais les indispensables contraintes liées à la pratique du jeu - celles que définissent, dans chaque cas, des « règles du jeu » plus ou moins exigeantes - ont une valeur heuristique. Obligeant à réfléchir d'une autre façon que dans l'exercice régulier de la recherche, elles peuvent conduire la pensée vers des voies imprévues, dans des directions que, peut-être à tort, le chercheur expérimenté renonce à explorer. Pierre Bayard en apporte régulièrement la preuve.

Par ailleurs, la «critique policière " semble retrouver des intuitions fondatrices de la démarche de certains historiens : ceux qui ont théorisé et pratiqué, en Italie d'abord, la micro-storia. Dans un article déjà ancien, qui faisait d'ailleurs référence à la naissance du roman policier à la fin du XIX ${ }^{e}$ siècle, Carlo Ginzburg demandait en effet aux historiens de s'identifier au détective en quête d'indices. En s'attachant plutôt aux détails qu'aux structures d'ensemble, il leur suggérait de retrouver la vérité d'une société disparue à partir de traces éparses ${ }^{2}$. L'attention portée par Pierre Bayard aux détails de l'œuvre, afin d'en délivrer un sens neuf, pourrait sembler relever d'une même logique. Une différence essentielle, néanmoins, sépare les deux démarches. 
Cette différence ne tient pas à la définition, assez peu enthousiasmante, que Pierre Bayard propose du métier d'historien. Selon lui, en effet, le " principe d'incomplétude » d'une œuvre littéraire, reposant sur le fait incontestable que l'auteur ne saurait nous dire absolument tout de l'univers qu'il a créé - que ses personnages, en particulier, ont inévitablement vécu d'autres expériences que celles qui sont rapportées dans le récit -, est très différent de l'incomplétude du récit historique :

Ces défaillances du monde de l'œuvre ne tiennent pas à un défaut d'information que le travail de recherche, comme en histoire, peut espérer combler un jour, mais à un défaut de structure. ${ }^{3}$

11 De la part d'un auteur si prompt à déjouer les routines de la pensée, une définition aussi positiviste de l'histoire déçoit un peu. Il est regrettablement réducteur d'estimer que l'essentiel du travail des historiens consiste d'abord en la découverte de nouvelles sources (même s'il ne s'agit pas de nier l'importance que de telles découvertes peuvent parfois occasionner). À cette conception un peu figée des études historiques, on préférera la leçon proposée plus loin par Pierre Bayard, fondatrice de la possibilité même de la « critique policière » et que l'auteur résume ainsi :

Nous ne lisons pas la même œuvre au fil du temps et c'est collectivement que nous sommes aujourd'hui sensibles à certains détails du texte qui frappent notre modernité et peuvent nous conduire, par le type de complément que nous lui apportons, à des approches renouvelées. ${ }^{4}$

On voit ici en quoi le rôle du détail chez Pierre Bayard diffère de celui que lui assigne Carlo Ginzburg. Là où la micro-storia continuait à privilégier la quête de sources inédites, se contentant d'élever dorénavant au statut de source nécessaire et suffisante des documents qui jusqu'alors ne semblaient renseigner que sur des détails de l'histoire, Pierre Bayard, en dépit de sa conception un peu rigide de la démarche historienne, suggère une dignité différente du détail. La valeur de ce dernier ne dépend plus de la capacité de l'historien à l'analyser après l'avoir exhumé des archives. À l'image de la lettre volée d'Edgar Allan Poe, le détail de Pierre Bayard a toujours été là, au vu et au su de tous. Et Pierre Bayard nous suggère que le bon historien est d'abord, tel le chevalier Dupin, celui qui saura, parce que son époque et sa personnalité le lui permettent, observer avec un regard neuf ce détail que tant d'autres avant lui ont déjà vu. Sans doute Pierre Bayard, prisonnier de sa conception réductrice du métier d'historien, ne le dit-il pas explicitement. Il ne le pense peut-être même pas mais, au nom de ses propres principes de méthode, il ne peut pas nous dénier le droit de lire son œuvre de cette façon. Ainsi n'est-ce pas le moindre des paradoxes de la "critique policière » que d'inviter les historiens à dépasser l'horizon positiviste en deçà duquel, pourtant, son fondateur semble vouloir les situer.

\section{NOTES}

1. Opinion apparemment partagée par Pierre Bayard lui-même. Voir Comment parler des livres que l'on n'a pas lus, Paris, Minuit, 2007, p. 73. 
2. Carlo Ginzburg, "Traces. Racines d'un paradigme indiciaire", dans Mythes, emblèmes, traces. Morphologie et histoire, Paris, Flammarion, 1988, p. 153.

3. Pierre Bayard, L'Affaire du chien des Baskerville, Paris, Minuit, 2008, p. 67.

4. Ibid., p. 70.

\section{AUTEURS}

\section{SYLVAIN VENAYRE}

Sylvain Venayre est maître de conférences en histoire contemporaine à l'université Paris IPanthéon-Sorbonne (Centre d'histoire du XIx siècle). Ses travaux portent sur l'histoire culturelle du voyage et sur les représentations du temps. Il a notamment publié La Gloire de l'aventure. Genèse d'une mystique moderne. 1850-1940 (Paris, Aubier, 2002) et dirigé plusieurs ouvrages collectifs dont, récemment, avec Nicolas Bourguinat, Voyager en Europe de Humboldt à Stendhal (Paris, Nouveau Monde, 2007). Il a également établi, en collaboration avec Jean-Philippe Stassen, une édition illustrée et commentée de Cour des ténèbres de Joseph Conrad (Paris, Futuropolis / Gallimard, 2007). 